Research Article

\title{
Secondary structure of nrDNA Internal Transcribed Spacers as a useful tool to align highly divergent species in phylogenetic studies
}

Giovanna C. Giudicelli ${ }^{1}$, Geraldo Mäder ${ }^{1}$, Gustavo A. Silva-Arias ${ }^{1}$, Priscilla M. Zamberlan ${ }^{1}$, Sandro L. Bonatto ${ }^{2}$ and Loreta B. Freitas ${ }^{1}$

${ }^{1}$ Laboratory of Molecular Evolution, Department of Genetics, Universidade Federal do Rio Grande do Sul (UFRGS), Porto Alegre, Brazil

${ }^{2}$ Laboratory of Genomic and Molecular Biology, Pontifícia Universidade Católica do Rio Grande do Sul, Porto Alegre, RS, Brazil

\begin{abstract}
Recently, it has been suggested that internal transcribed spacer (ITS) sequences are under selective constraints to preserve their secondary structure. Here, we investigate the patterns of the ITS nucleotide and secondary structure conservation across the Passiflora L. genus to evaluate the potential use of secondary structure data as a helpful tool for the alignment in taxonomically complex genera. Considering the frequent use of ITS, this study also presents a perspective on future analyses in other plant groups. The ITS1 and ITS2 sequences presented significant differences for mean values of the lowest energy state (LES) and for number of hairpins in different Passiflora subgenera. Statistical analyses for the subgenera separately support significant differences between the LES values and the total number of secondary structures for ITS. In order to evaluate whether the LES values of ITS secondary structures were related to selective constraints, we compared these results among 120 ITS sequences from Passiflora species and 120 randomly generated sequences. These analyses indicated that Passiflora ITS sequences present characteristics of a region under selective constraint to maintain the secondary structure showing to be a promising tool to improve the alignments and identify sites with non-neutral substitutions or those correlated evolutionary steps.
\end{abstract}

Keywords: Passiflora; lowest energy state; hairpins; ITS1; ITS2.

Received: February 24, 2016; Accepted: August 23, 2016.

\section{Introduction}

Ribosomal DNA (rDNA) consists of one of the largest multigenic families in eukaryotic genomes and is present at one or several locations in arrays of tandem elements (Hillis and Dixon, 1991). Each unit is composed of three rRNA gene regions $(18 \mathrm{~S}, 5.8 \mathrm{~S}$, and $26 \mathrm{~S}$ ) that are preceded by an external transcribed spacer, and the two internal transcribed spacers ITS1 and ITS2 separate the genes respectively (Buckler-IV et al., 1997). The rDNA exons are highly conserved across eukaryotic organisms, whereas the ITS regions present length variability due to point mutations and insertions/deletions (indels) (Baldwin et al., 1995; Álvarez and Wendel, 2003). Gene regions are further processed to produce the mature RNAs to form the cytoplasmic ribosomes, whereas ITS regions suffer a specific cleavage during the maturation of the ribosomal subunits and are thus not incorporated into mature ribosomes (Hillis and Dixon, 1991; Baldwin et al., 1995). ITS regions play an im-

Send corresponding to Loreta B. Freitas. Laboratory of Molecular Evolution, Department of Genetics, Universidade Federal do Rio Grande do Sul, P.O. Box 15053, 91501-970, Porto Alegre, RS, Brazil. E-mail: loreta.freitas@ufrgs.br portant role in rRNA processing. Deletion of the central portion of ITS1, including a processing region that is used in the early stages of ribosomal maturation, blocks the formation of 40S subunits (Musters et al., 1990), whereas small deletions in the 5'-terminal region of ITS2 prevent the maturation of $26 \mathrm{~S}$ rRNA, and deletions in the 3 '-terminal portion severely reduce the efficiency of this process (van der Sande et al., 1992). The maturation and splicing processes depend on the secondary structure of ITS regions and thus imply some degree of conservation at the sequence or structural level (Mai and Coleman, 1997).

ITS sequences have been widely used in the inference of phylogenetic hypotheses or in molecular evolution studies in plants (Álvarez and Wendel, 2003), including several taxonomic levels (Kress et al., 2002; Zimmer et al., 2002; Koch et al., 2003; Rauscher et al., 2004; Bellarosa et al., 2005; Kan et al., 2007; Queiroz et al., 2011; Ghada et al., 2013; Fan et al., 2014). In the Passiflora genus, ITS regions are the most commonly used marker for estimating the phylogenetic relationships among subgenera and species (Muschner et al., 2003; Krosnick and Freudenstein, 2005; Lorenz-Lemke et al., 2005; Koehler-Santos et al., 2006; 
Mäder et al., 2010; Cazé et al., 2013; Krosnick et al., 2013; Ramaiya et al., 2014), although this is usually combined with other sequences in these studies.

The Passiflora genus is the largest member of the Passifloraceae family, with more than 520 species widely distributed in the Neotropical region and a few species occurring in the Old World (Deginani, 2001; Ulmer and MacDougal, 2004). This genus was initially divided into 22 or 23 subgenera based on floral morphology (Killip, 1938; Escobar, 1989), but the current infrageneric classification (Feuillet and MacDougal, 2003) regrouped the species into four subgenera that have been partially or fully corroborated in phylogenetic studies (Muschner et al., 2003, 2012; Yockteng and Nadot 2004a; Hansen et al., 2006, 2007): Astrophea (DC.) Mast., Decaloba (DC.) Rchb., Deidamioides (Harms) Killip, and Passiflora. The species of the Passiflora genus present a large diversity of floral and vegetative features, which contributes to the complex taxonomy of this group (Krosnick and Freudenstein, 2005).

The first study to obtain a molecular phylogeny for Passiflora (Muschner et al., 2003) described a high divergence among ITS sequences of the different subgenera; consequently, alignment of these sequences presents several ambiguous regions and indels. For that reason, authors suggested a separate alignment per subgenus. Several plastid regions (Muschner et al., 2003; Krosnick and Freudenstein, 2005; Hansen et al., 2006) and other nuclear markers (Yockteng and Nadot, 2004a,b; Muschner et al., 2012) have also been used in Passiflora studies; however, they do not present sufficient variability to distinguish species as efficiently as observed in ITS. Despite this, the low variability in plastid and other nuclear regions is advantageous in relation to ITS because allows the alignment of all subgenera simultaneously (Muschner et al., 2003; Mäder et al., 2010).

Mutations in the tandem repeats of rDNA are commonly homogenized through concerted evolution (Arnheim et al., 1980; Baldwin et al., 1995). This phenomenon makes each copy of an rRNA array very similar to other copies within individuals and species, thus providing a high degree of similarity in gene family and affecting the region variability (Hillis and Dixon, 1991; Buckler-IV et al., 1997). However, the high level of ITS polymorphism present in Passiflora species indicates that the process of concerted evolution is not sufficiently fast to homogenize the region (Lorenz-Lemke et al., 2005; Koehler-Santos et al., 2006), as observed in other plant groups (Wissemann and Ritz, 2005; Harpke and Peterson, 2006). Melo and Guerra (2003) suggested that the high variability of ITS present in Passiflora could be related to the chromosomal location of rDNA in this genus, as has been suggested for Gossypium L. (Wendel et al., 1995), because the 45S sites where the ITS region is located were mapped in subterminal position, which interferes with copy homogenization (Li and Zhang, 2002).
The presence of a high number of polymorphisms in sequences makes it more difficult to obtain good alignments, and this could affect phylogenetic inference (Álvarez and Wendel, 2003). For that reason, the aim of this study was to investigate the patterns of ITS nucleotide and secondary structure conservation across the Passiflora genus to evaluate the potential use of secondary structure data as a helpful tool to improve the alignments in a genus that presents high taxonomic complexity. We also evaluated for the first time whether the ITS sequences are under selective constraints, considering their secondary structure in Passiflora species. Considering the frequent use of ITS sequences, this study also presents a perspective for future analyses of secondary structure and estimates of phylogenies in other plant groups. Moreover, this original contribution improves the database of ITS secondary structure that is essential for a trustworthy reference to phylogenetic and DNA barcoding analyses (Ankenbrand et al., 2015).

\section{Material and Methods}

\section{Taxon sampling}

GenBank sampling included 870 accessions of ITS1 and ITS2 from 163 species representatives of all four subgenera: Astrophea (9 spp.), Decaloba (112 spp.), Deidamioides (6 spp.), and Passiflora (36 spp.). We also included 74 new sequences (GenBank accessions numbers: KP769869- KP769905; KP769917- KP769953) from 26 species (Astrophea 5 spp., Decaloba 1 spp., and Passiflora 20 spp.), totaling 944 sequences from 189 Passiflora species. All these sequences were used to assess diversity in Passiflora. Subgenera were represented by different number of sequences according the number of species included on each one. Sequences that presented a large number of missing data in initial or final portions of ITS1 or ITS2 were not included in our analyses.

\section{Plant material, DNA extraction, PCR amplification and sequencing}

To obtain the new sequences, the total DNA was extracted from young leaves dried in silica gel using the method of Roy et al. (1992). Voucher specimens were deposited at ICN Herbarium (Department of Botany, Federal University of Rio Grande do Sul, Porto Alegre, Brazil). ITS1 and ITS2 regions were amplified using primers 92 and 75 (White et al., 1990) and amplification conditions as previously described (Desfeux and Lejeune, 1996). To exclude the presence of low stability templates, we used $10 \%$ dimethyl sulfoxide (DMSO) (Buckler-IV et al., 1997; Fuertes-Aguilar et al., 1999). We checked the quality and quantity of PCR products by horizontal electrophoresis in $1 \%$ agarose gel stained with GelRed (Biotium, UK), and purified them using the polyethyleneglycol (PEG) precipitation method (Dunn and Blattner, 1987). We performed the sequencing reactions automatically in a MegaBACE 
1000 DNA Analysis System (GE Healthcare Biosciences, Pittsburgh, PA, USA).

\section{Data analyses}

We removed the 5.8S gene region from ITS sequences because of its well-conserved nature, and ITS1 and ITS2 were analyzed individually. We discarded identical sequences obtained from a same species, such that each sequence type was considered only once, using DnaSP 5 (Librado and Rojas, 2009). Because Passiflora subgenera present high genetic variability among them, we conducted the analysis considering each subgenus separately. Consequently, for each subgenus, sequences of ITS1 or ITS2 were automatically aligned using default parameters in ClustalX (Thompson et al., 1997), visually reviewed, and manually adjusted using MEGA6 (Tamura et al., 2013). We deleted ambiguous sites from ITS sequences (Mäder $e t$ al., 2010), and gaps were coded as binary characters (Simmons and Ochoterena 2000) using GapCoder (Young and Healy, 2003).

We selected 120 sequences from 60 species (Astrophea 6 ssp., Decaloba 39 ssp., Deidamioides 6 ssp., and Passiflora $9 \mathrm{ssp}$.) to model secondary structures of both ITS1 and ITS2 regions. For this selection, we first constructed phylogenetic trees in BEAST 1.8 (Drummond et al., 2012) using the HKY substitution model with four gamma categories, a Yule tree prior, and $10^{7}$ chain lengths. The first 1000 trees were discarded as Òburn inÓ. We used the JModelTest software (Guindon and Gascuel, 2003; Darriba et al., 2012) to select the best evolution model for our BEAST analysis, but because the resulting models were not available in the BEAST software, we selected the HKY substitution model as the closest to our results, based on Passiflora phylogenies previously obtained (Muschner et al., 2003; Lorenz-Lemke et al., 2005; Koehler-Santos et al., 2006; Mäder et al., 2010; Cazé et al., 2013). Phylogenetic trees obtained using BEAST are available in Supplementary Material (Figures S1-S8). We selected the species to obtain the ITS1 and ITS2 secondary structures considering only species from well-supported clades (posterior probability 30.7 ). We included a greater number of Decaloba species to test differences between secondary structures of species from different supersections that are phylogenetically well supported (Krosnick et al., 2013). For this analysis, we randomly chose three species from each supersection, except for Multiflora: all sequences available for this supersection were analyzed because it is the only paraphyletic supersection in Decaloba subgenus studies (Krosnick et al., 2013).

We calculate mean guanine-cytosine (GC) content for each Passiflora subgenus and ITS segment (ITS1 or ITS2) separately. Considering these values and the size variation (bp) of the original ITS sequences, we generated 120 random sequences to evaluate whether the total number of secondary structures and lowest energy state (LES) values of ITS1 and ITS2 sequences were related to selective constraints. These sequences were obtained using a Random DNA Sequence Generator (www.faculty.ucr.edu/ $\sim$ mmaduro/random.htm) and presented the same length range (176 bp to $280 \mathrm{bp}$ ) of the original sequences. Therefore, for each Passiflora ITS1 or ITS2 sequence, we obtained a random sequence that presented the same size (bp) and a similar GC content. All secondary structures for those random sequences were also modeled, and the obtained information is available in Supplementary Material Table S2.

\section{Secondary structure prediction}

We modeled ITS1 and ITS2 putative secondary structures for the 120 sequences of 60 Passiflora species and 120 random sequences using RNAstructure 5.3 (Reuster and Mathew, 2010). Output parameters of this software included the LES and total number of structures for each analyzed sequence. We manually analyzed the number of hairpins and paired nucleotides of ITS sequences as performed by Edger et al. (2014). Our analysis included for each Passiflora subgenus and random sequences: list of species, GenBank accession numbers, lengths, LES, total number of structures, number of hairpins, and number and percentage of paired nucleotides (Supplementary Material Tables S1 and S2).

We use the Wilcoxon-Mann-Whitney test in R software package (R Development Core Team, 2011) to assess whether there are significant differences between secondary structure parameters (i.e., LES, total number of structures, and number of hairpins) obtained for ITS1, ITS2, and random sequences. We performed an analysis comparing all ITS1 and ITS2 sequences to the 120 random sequences generated and another two analyses considering only ITS1 or ITS2 sequences and their 60 random sequences separately. We assessed differences for the same secondary structure parameters among the four Passiflora subgenera using a Kruskal-Wallis test (Kruskal and Wallis, 1952).

\section{Results and Discussion}

\section{ITS1 and ITS2 sequences characteristics and secondary structure}

Alignments for all subgenera presented indels with different extensions among species, as previously observed in other Passiflora studies (Muschner et al., 2003; Mäder et al., 2010). Characteristics of ITS1 and ITS2 regions evaluated per subgenera are summarized in Table 1. ITS1 sequences always presented higher length than the ITS2 sequences within each subgenus. Decaloba showed the longest length for ITS1 (336 base pairs - bp), as did Passiflora for ITS2 (239 bp). Decaloba exhibited the highest percentages of variable and informative sites for both ITS1 and ITS2 sequences, whereas Deidamioides showed the lowest percentages of variable and informative sites for ITS1 and Astrophea for ITS2 sequences. 
Table 1 - Characteristics of ITS1 and ITS2 dataset presented per Passiflora subgenera.

\begin{tabular}{|c|c|c|c|c|c|c|}
\hline Subgenus & ITS region & $\mathrm{N}$ individuals & $\mathrm{N}$ species & Alignment length (bp) & Variable characters $(\%)$ & PI characters $(\%)$ \\
\hline \multirow[t]{2}{*}{ Astrophea } & ITS1 & 26 & 14 & 288 & 31.25 & 20.14 \\
\hline & ITS2 & 31 & 14 & 235 & 28.51 & 16.17 \\
\hline \multirow[t]{2}{*}{ Decaloba } & ITS1 & 202 & 113 & 336 & 71.43 & 58.93 \\
\hline & ITS2 & 180 & 113 & 231 & 66.23 & 53.25 \\
\hline \multirow[t]{2}{*}{ Deidamioides } & ITS1 & 70 & 6 & 288 & 27.43 & 18.06 \\
\hline & ITS2 & 59 & 6 & 226 & 36.28 & 23.01 \\
\hline \multirow[t]{2}{*}{ Passiflora } & ITS1 & 126 & 56 & 260 & 46.54 & 31.54 \\
\hline & ITS2 & 106 & 56 & 239 & 46.86 & 25.94 \\
\hline
\end{tabular}

ITS, internal transcribed spacer; BP, base pairs; PI, parsimony informative

Considering ITS1 and ITS2 sequences of all subgenera, the ITS1 region size range was between 220 and 280 bp, whereas ITS2 ranged between 176 and $222 \mathrm{bp}$. We evaluated each ITS1 and ITS2 sequence based on different parameters: sequence length, LES, total number of structures, number of hairpins, and number and percentage of paired nucleotides (Table 2; Supplementary Material Table S1). ITS1 sequences presented higher average length (mean 263 bp, Standard Deviation SD = 19 bp) than ITS2 sequences (mean $206 \mathrm{bp}$, SD $7 \mathrm{bp}$ ), considering the four subgenera. Additionally, ITS1 sequences displayed significant differences and lower mean values of LES than ITS2 sequences (-105.6 and -80.5 degrees, respectively; $P<0.001$; Table 2). This result means that although they are of shorter sequence length, ITS2 sequences require more energy than ITS1 to form the secondary structures.

ITS1 sequences exhibited higher values of variable and informative characters, whereas the ITS2 region showed higher numbers of conserved sites, as previously observed for a different Passiflora species set (Giudicelli et al., 2015). High conservative patterns in ITS2 sequences have been previously reported and related to structural constraints that are present at very deep phylogenetic scales in eukaryotes (Mai and Coleman 1997; Shultz et al., 2005). Although highly conserved motifs have also been reported for ITS1 sequences in plants, such motifs seem to be shorter than those observed in ITS2, and in Passiflora ITS1 region, remaining parts of the sequence are more variable these motifs (Liu and Schardl 1994).

No statistically significant differences were observed between ITS1 and ITS2 in the total number of possible secondary structures. ITS1 and ITS2 sequences exhibited statistically significant differences in the numbers of hairpins $(P<0.001$; Table 2; Figure 1). The most frequent number of hairpins for ITS1 was seven $(\min =2, \max =8)$, whereas the most frequent number of hairpins in ITS2 was three

Table 2 - Mean parameters values analyzed for ITS1 and ITS2 sequences per Passiflora subgenera. Standard deviations for each value are shown in parentheses.

\begin{tabular}{|c|c|c|c|c|c|}
\hline \multicolumn{6}{|c|}{ ITS 1} \\
\hline & Astrophea $[6]^{*}$ & Decaloba $[39]^{*}$ & Deidamioides [6]* & Passiflora [9]* & All sequences $[60]^{*}$ \\
\hline Length (bp) & $270.2(2.3)$ & $271.8(8.8)$ & $251.2(19.7)$ & $225.8(2.5)$ & $262.7(19.1)$ \\
\hline Lowest Energy State (LES) & $-121.8(5.5)$ & $-104.2(9.4)$ & $-104.4(4.9)$ & $-101.8(6.1)$ & $-105.6(9.8)$ \\
\hline Total number of Structures & $15.8(3.9)$ & $12.6(4.1)$ & $16(4.5)$ & $8.8(3.8)$ & $12.7(4.5)$ \\
\hline No. Hairpins & $5.8(1.6)$ & $5.6(1.4)$ & $5.2(2.1)$ & $4.0(0.9)$ & $5.3(1.5)$ \\
\hline No. Paired Nucleotides & $168.3(2.7)$ & $167.7(10.8)$ & $149.3(12.6)$ & $135.3(7.3)$ & $161.1(15.7)$ \\
\hline$\%$ Paired Nucleotides & $62.3(0.8)$ & $61.7(2.8)$ & $59.4(0.5)$ & $59.9(3.1)$ & $61.3(2.7)$ \\
\hline \multicolumn{6}{|c|}{ ITS 2} \\
\hline Length (bp) & $208.8(7.3)$ & $208.1(3.2)$ & $204.5(5)$ & $195.3(10.9)$ & $205.9(7.1)$ \\
\hline Lowest Energy State (LES) & $-90.3(1.5)$ & $-74.9(6.2)$ & $-87.9(7.9)$ & $-92.9(6.1)$ & $-80.4(9.7)$ \\
\hline Total number of Structures & $14.2(2.2)$ & $11.2(4)$ & $13.8(3.6)$ & $17.3(3.8)$ & $12.7(4.3)$ \\
\hline No. Hairpins & $2.8(1.3)$ & $3.3(0.6)$ & $2.3(0.8)$ & $2.8(1)$ & $3.0(0.8)$ \\
\hline No. Paired Nucleotides & $128.7(4.5)$ & $123.4(6.1)$ & $128.7(4.1)$ & $130.7(10.5)$ & $125.5(7.1)$ \\
\hline$\%$ Paired Nucleotides & $61.6(1)$ & $59.3(3)$ & $62.9(2.6)$ & $66.8(2.6)$ & $61.0(3.8)$ \\
\hline
\end{tabular}

*Numbers of analyzed sequences. ITS, internal transcribed spacer; BP, base pairs 
a) $\stackrel{\text { o }}{2}$

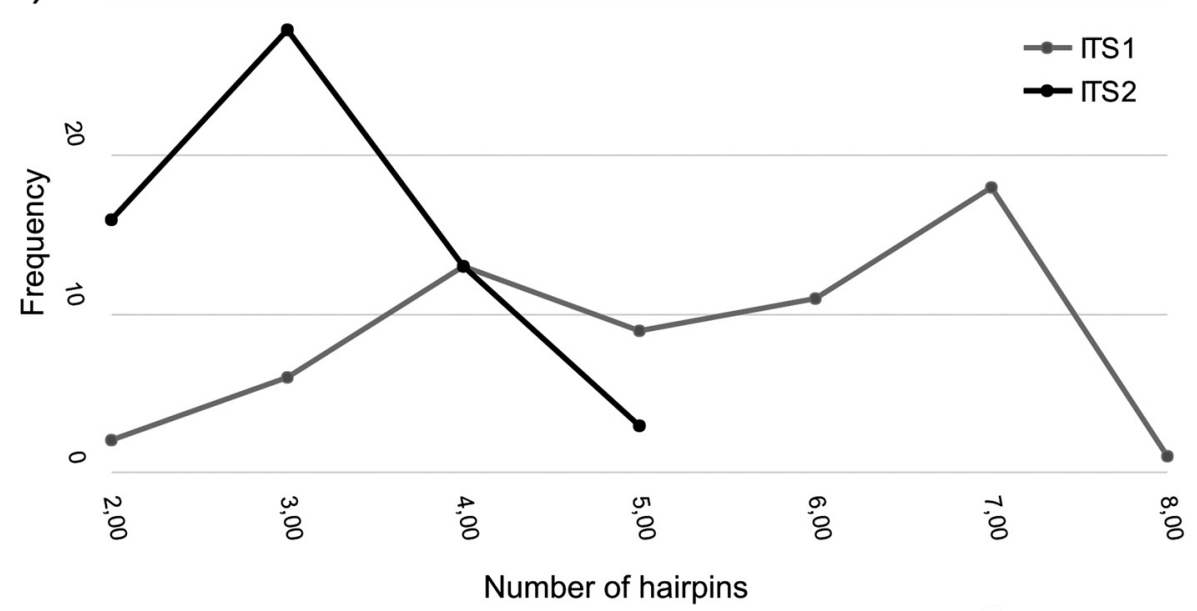

b)

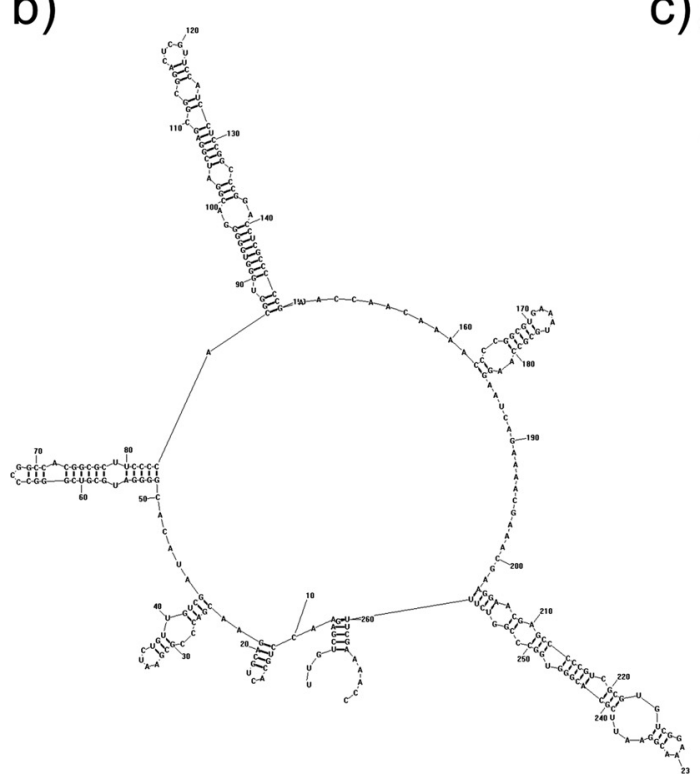

c)

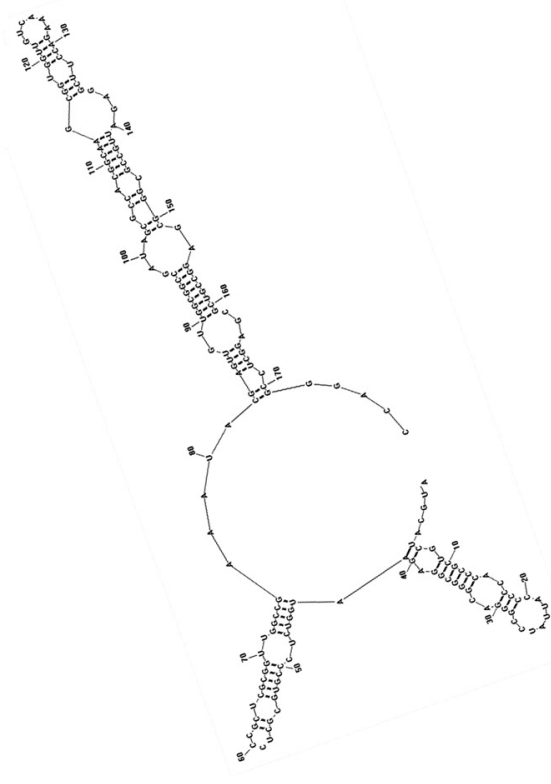

Figure 1 - Frequency and examples of number of hairpins for ITS1 and ITS2 sequences. (a) Frequency of the total number of hairpins for ITS1 (gray) and ITS2 (black) sequences. Secondary structures examples with (b) seven hairpins in ITS1 and (c) three hairpins in ITS2.

$(\min =2, \max =5)$. No differences were observed in the percentage of paired nucleotides for ITS1 and ITS2 sequences (Table 2). Sequences had over $50 \%$ of their bases paired with other nucleotides to form secondary structures (Table 2), as observed for Brassicaceae (Edger et al., 2014). Because ITS1 sequences displayed longer lengths than ITS2 and because the sequences exhibited significant differences according to the number of paired nucleotides, ITS1 presented a higher number of paired nucleotides $(P<0.001$; Table 2).

Statistical analyses conducted for the four subgenera separately support significant differences between LES values for ITS1 $(P<0.01)$ and ITS2 $(P<0.001)$ sequences. These differences are not surprising when taking into account the large observed differences of the sequences among the four Passiflora subgenera. The highest mean value of LES for ITS1 was observed in Passiflora subgenus (-101.8 degrees), and for ITS2 in Decaloba (-74.9 degrees; Table 2). There were also statistically significant differences in the total number of possible secondary structures among subgenera for ITS1 $(P<0.01)$ and ITS2 $(P<0.01)$. For ITS1, Deidamioides showed a higher mean value of possible alternate secondary structures (16 possible structures, Table 2), while for ITS2 this was the case for Passiflora (17 possible structures; Table 2). No statistically significant differences were observed within Decaloba when the species were separated according to the supersections previously proposed (Feuillet and MacDougal, 2003; Ulmer and MacDougal, 2004). We also observed that different sequences types of a species did not exhibit differences on their secondary structure (data not shown). 
Comparison of secondary structures of ITS1, ITS2, and random sequences to assess selective constraints of ITS regions

Randomly generated sequences presented -80.2 degrees for LES $(\min =-126.7, \max =-53.9)$ on average and a mean of 17.2 possible secondary structures $(\min =3$, $\max =20$ ). In all three analyses comparing ITS and randomly-generated sequences (Figure 2), we found different values: to combined ITS sequences, LES $P<0.001$ and number of possible secondary structures $P<0.001$; ITS1 only, LES $P<0.001$ and number of possible secondary structures $P<0.001$; and ITS2 only, LES $P<0.001$ and number of possible secondary structures $P<0.001$. Those analyses indicated that Passiflora sequences secondary structures exhibit significantly lower energy states and less structures compared with randomly generated sequences that are similar in length and GC content. These results suggest that Passiflora ITS sequences show characteristics of a region that is under selective constraint to maintain its secondary structure.

Different secondary structure predictor softwares may propose very distinct structures from the same sequence, even when using the same initial parameters (Gottschling and Pltner, 2004). Additionally, thermodynamically optimal structures certainly do not reflect the structures in cells, because there are many unaccounted biochemical factors that give a dynamic (temporarily unstable) characteristic to the secondary structure of the rRNA transcripts. However, in addition to the strong differences seen among the Passiflora sequences, there are high- ly conserved structural patterns and sequence motifs for the entire genus, which support the suggestion of selective constraints for maintaining the secondary structures.

\section{Utility of ITS secondary structures to improve alignments}

Analyses conducted for the four Passiflora subgenera separately revealed no statistically significant differences in the total number of hairpins among subgenera. However, although no pattern was observed to characterize each subgenus, secondary structures were shown to be a potential tool for improving the quality of alignments and identifying possible sites with non-neutral substitution patterns or sites with correlated evolution. This allows to obtain better results in phylogenetic inferences for Passiflora, as previously observed for other plant groups (Gottschling et al., 2001; Goertzen et al., 2003).

The highly conserved motif in the central region of the ITS1 sequence previously described for flowering plants (Liu and Schardl, 1994) was also present in the Passiflora sequences and was helpful in aligning the Passiflora sequences for phylogenetic analysis. Other ITS1 conserved motifs described in other groups were also found in Passiflora, such as 5'AAGGAA 3' in the central region of ITS1 (Liu and Schardl, 1994; Gottschling et al., 2001) and a region rich in adenine and cytosine (Coleman et al., 1998). Conserved patterns that had already been described in angiosperms (Hershkovitz and Zimmer 1996; Mai and Coleman, 1997) and green algae (Mai and Coleman, 1997) were also observed in Passiflora ITS2 sequences. These

\section{ITS Sequences Compared to Random Sequences}

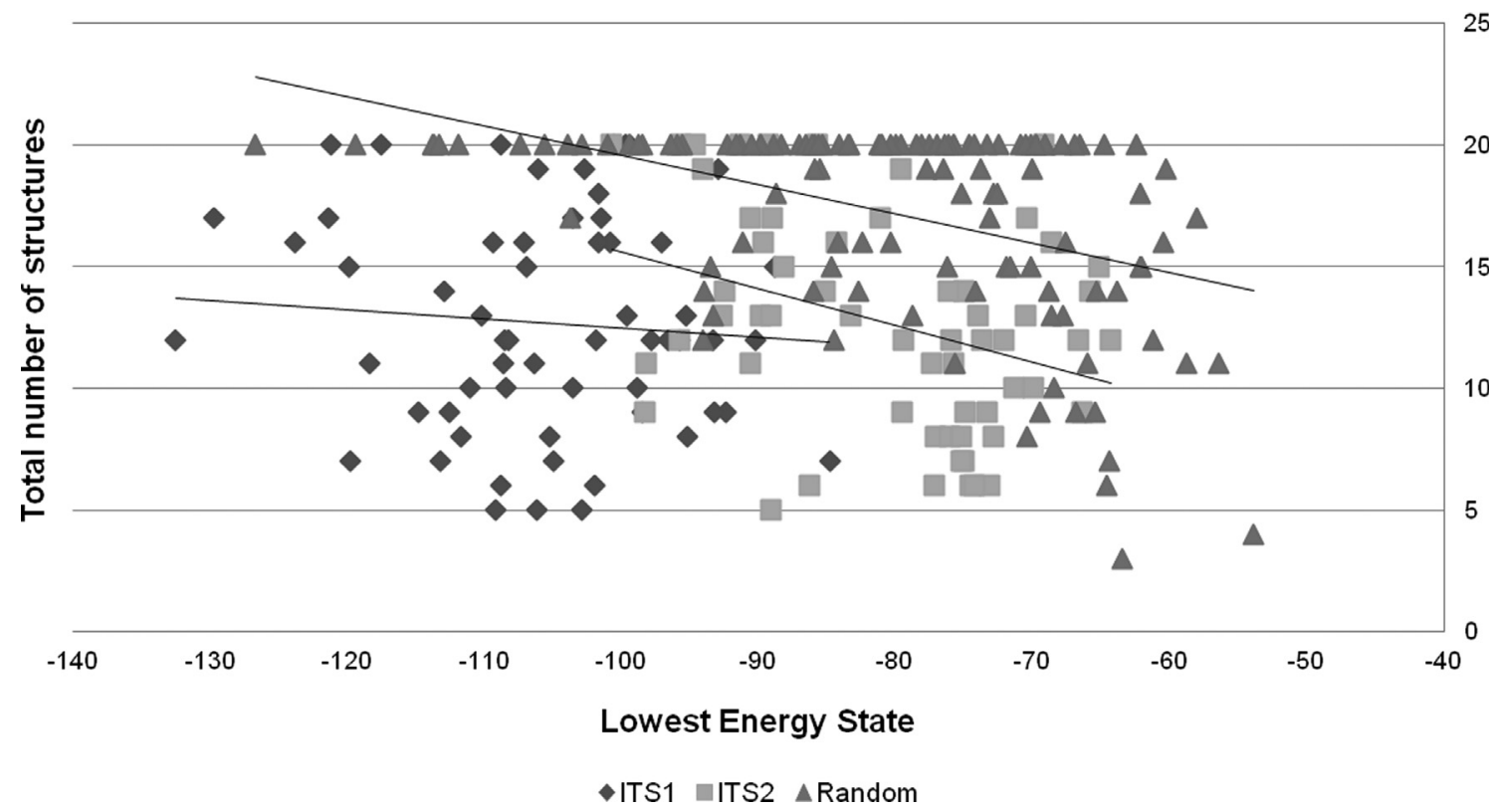

Figure 2 - ITS sequences compared with random sequences. Scatter plot of t lowest energy state values (X-axis) and total number of secondary structures (Y-axis). Squares represent ITS1 sequences; circles represent ITS2 sequences; and triangles represent randomly generated sequences. 
conserved motifs are commonly associated with the formation of hairpins in secondary structure.

Comparisons among species from different subgenera that presented the same number of hairpins showed conserved patterns on secondary structure that could be useful for improving the Passiflora sequence alignments. For example, when comparing the ITS1 region among species from the Astrophea, Decaloba, and Deidamioides subgenera with seven hairpins, we observed a common hairpin with 5-6 paired bases formed by initial (5'-TCGAA) and final (TTCGA-3') portions. This same motif was also found in ITS1 sequences that presented different numbers of hairpins, thus demonstrating the conserved nature of ITS1 sequences and serving as a reason why a universal set of primers could be used to amplify sequences of plants and fungi (White et al., 1990; Álvarez and Wendel, 2003). The analysis of secondary structure also facilitates the identification of indels when sequences with different number of hairpins are compared, thus contributing to the improvement of the alignment of sequences with different lengths and high diversity index.

The conservation of specific nucleotides in secondary structures contributes to positional homology and is a helpful tool to improve the alignments (Keller et al., 2010). Therefore, we believe that analyses of ITS secondary structures obtained from the Passiflora species could be a valuable source of information to improve alignment, considering the difficulties of aligning species from the different subgenera (Muschner et al., 2003; Mäder et al., 2010), and the same strategy could be used in other plant genera that present a complex taxonomy.

Many Passiflora studies have used ITS sequences to estimate phylogenetic relationships, and our results show that ITS regions are under selective constraints to maintain their secondary structures, likely due to their specific functions in the splicing process, as was observed for Brassicaceae (Edger et al., 2014). The use of ITS as a molecular marker carries some risk and must be done cautiously, but it presents the advantage of having a great deal of sequence data for this locus (Krosnick et al., 2013). Plastid markers are not sufficiently variable in the Passiflora genus (Muschner et al., 2003; Mäder et al., 2010; Cazé et al., 2013), and ITS secondary structures could thus be a helpful tool to improve phylogenetic inferences and evolutionary studies in this genus and others.

\section{Acknowledgments}

This work was supported by the Conselho Nacional de Desenvolvimento Científico e Tecnológico (CNPq), the Coordenação de Aperfeiçoamento de Pessoal de Nível Superior (CAPES), and the Programa de Pós-Graduação em Genética e Biologia Molecular da Universidade Federal do Rio Grande do Sul (PPGBM-UFRGS).

\section{References}

Ankenbrand MJ, Keller A, Wolf M, Schultz J and Frster F (2015) ITS2 database V: Twice as much. Mol Biol Evol 32:3030-3032.

Álvarez I and Wendel JF (2003) Ribosomal ITS sequences and plant phylogenetic inference. Mol Phylogenet Evol 29:417-434.

Arnheim N, Krystal M, Schmickel R, Wilson G, Ryder O and Zimmer E (1980) Molecular evidence for genetic exchanges among ribosomal genes on non-homologous chromosomes in man and apes. Proc Natl Acad Sci USA 77:7323-7327.

Baldwin BG, Sanderson MJ, Porter JM, Wojciechowski MF, Campbell CS and Donoghue MJ (1995) The ITS region of nuclear ribosomal DNA: A valuable source of evidence on angiosperm phylogeny. Ann Mo Bot Gard 82:247-277.

Bellarosa R, Simeonea MC, Papinib A and Schironea B (2005) Utility of ITS sequence data for phylogenetic reconstruction of Italian Quercus spp. Mol Phylogenet Evol 34:355-370.

Buckler-IV ES, Ippolito A and Holtsford TP (1997) The evolution of ribosomal DNA: Divergent paralogues and phylogenetic implications. Genetics 145:821-832.

Cazé ALR, Mäder G, Bonatto SL and Freitas LB (2013) A molecular systematic analysis of Passiflora ovalis and Passiflora contracta (Passifloraceae). Phytotaxa 132:39-46.

Coleman AW, Maria Preparata R, Mehrota B and Mai JC (1998) Derivation of the secondary structure of the ITS-1 transcript in Volvocales and its taxonomic correlations. Protist 149:135-146.

Darriba D, Taboada GL, Doallo R and Posada D (2012) jModelTest 2: More models, new heuristics and parallel computing. Nat Methods 9:772.

Deginani NB (2001) Las especies argentinas del género Passiflora (Passifloraceae). Darwiniana 39:43-129.

Desfeux C and Lejeune B (1996) Systematics of Euromediterranean Silene (Caryophyllaceae): Evidence from a phylogenetic analysis using ITS sequence. C R Acad Sci III 319:351-358.

Drummond AJ, Suchard MA, Xie D and Rambaut A (2012) Bayesian phylogenetics with 346 BEAUti and the BEAST 1.7. Mol Biol Evol 29:1969-1973.

Dunn IS and Blattner FR (1987) Charons 36 to 40: Multi-enzyme, high capacity, recombination deficient replacement vectors with polylinkers and polystuffers. Nucleic Acids Res 15:2677-2698.

Edger PP, Tang M, Bird KA, Mayfield DR, Conant G, Mummenhoff K, Koch MA and Pires JC (2014) Secondary structure analyses of the nuclear rRNA internal transcribed spacers and assessment of its phylogenetic utility across the Brassicaceae (Mustards). PLoS ONE 9:e101341.

Escobar LK (1989) A new subgenus and five new species in Passiflora (Passifloraceae) from South America. Ann Mo Bot Gard 76:877-885.

Fan X, Liu J, Sha LN, Sun GL, Hu ZQ, Zeng J, Kang HY, Zhang HQ, Wang Y, Wang XL, et al. (2014) Evolutionary pattern of rDNA following polyploidy in Leymus (Triticeae: Poaceae). Mol Phylogenet Evol 77:296-306.

Feuillet C and MacDougal JM (2003) A new infrageneric classification of Passiflora L. (Passifloraceae). Passiflora 13:34-38.

Fuertes-Aguilar J, Rosselló JA and Nieto-Feliner G (1999) Nuclear ribosomal DNA (nrDNA) concerted evolution in natu- 
ral and artificial hybrids of Armeria (Plumbaginaceae). Mol Ecol 8:1341-1346.

Ghada B, Ahmed BA, Messaoud M and Amel SH (2013) Genetic diversity and molecular evolution of the internal transcribed spacer (ITSs) of nuclear ribosomal DNA in the Tunisian fig cultivars (Ficus carica L.; Moracea). Biochem Syst Ecol 48:20-33.

Giudicelli GC, Mäder G and Freitas LB (2015) Efficiency of ITS sequences for DNA barcoding in Passiflora (Passifloraceae). Int J Mol Sci 16:7289-7303.

Goertzen LR, Cannone JJ, Gutell RR and Jansen RK (2003) ITS secondary structure derived from comparative analysis: Implications for sequence alignment and phylogeny of the Asteraceae. Mol Phylogenet Evol 29:216-234.

Gottschling M and Pltner J (2004) Secondary structure models of the nuclear internal transcribed spacer regions and $5.8 \mathrm{~S}$ rRNA in Calciodinelloideae (Peridiniaceae) and other dinoflagellates. Nucleic Acids Res 32:307-315.

Gottschling M, Hilger HH, Wolf M and Diane N (2001) Secondary structure of the ITS1 and its application in a reconstruction of the phylogeny of Boraginales. Plant Biol 3:629-636.

Guindon S and Gascuel O (2003) A simple, fast and accurate method to estimate large phylogenies by maximumlikelihood. Syst Biol 52:696-704.

Hansen AK, Gilbert LE, Simpson BB, Downie SR, Cervi AC and Jansen RK (2006) Phylogenetic relationships and chromosome number evolution in Passiflora. Syst Bot 31:138-150.

Hansen AK, Escobar LK, Gilbert LE and Jansen RK (2007) Paternal, maternal, and biparental inheritance of the chloroplast genome in Passiflora (Passifloraceae): Implications for phylogenetic studies. Am J Bot 94:42-46.

Harpke D and Peterson A (2006) Non-concerted ITS evolution in Mammillaria (Cactaceae). Mol Phylogenet Evol 41:579-593.

Hershkovitz MA and Zimmer EA (1996) Conservation patterns in angiosperm rDNA ITS2 sequences. Nucleic Acids Res 24:2857-2867.

Hillis DM and Dixon MT (1991) Ribosomal DNA: Molecular evolution and phylogenetic inference. Q Rev Biol 66:411-453.

Kan XZ, Wang SS, Ding X and Wang XQ (2007) Structural evolution of nrDNA ITS in Pinaceae and its phylogenetic implications. Mol Phylogenet Evol 44:765-777.

Keller A, Frster F, Müller T, Dandekar T, Schultz J and Wolf M (2010) Including RNA secondary structures improves accuracy and robustness in reconstruction of phylogenetic trees. Biol Direct 5:4.

Killip EP (1938) The American Species of Passifloraceae. Botanical Series, Field Museum of Natural History, Chicago, 613 p.

Koch MA, Dopes C and Mitchell-Olds T (2003) Multiple hybrid formation in natural populations: Concerted evolution of the Internal Transcribed Spacer of nuclear ribosomal DNA (ITS) in North American Arabis divaricarpa (Brassicaceae). Mol Biol Evol 20:338-350.

Koehler-Santos P, Lorenz-Lemke A, Muschner VC, Bonatto SL, Salzano FM and Freitas LB (2006) Molecular genetic variation in Passiflora alata (Passifloraceae), an invasive species in southern Brazil. Biol J Linn Soc Lond 88:611-630.
Kress WJ, Prince LM and Williams KJ (2002) The phylogeny and a new classification of the gingers (Zingiberaceae): Evidence from molecular data. Am J Bot 89:1682-1696.

Krosnick SE and Freudenstein JV (2005) Monophyly and floral character homology of Old World Passiflora (Subgenus Decaloba: Supersection Disemma). Syst Bot 30:139-152.

Krosnick SE, Porter-Utley KE, MacDougal JM, Jørgensen PM and McDade LA (2013) New insights into the evolution of Passiflora subgenus Decaloba (Passifloraceae): Phylogenetic relationships and morphological synapomorphies. Syst Bot 38:692-713.

Kruskal WH and Wallis WA (1952) Use of ranks in one-criterion variance analysis. J Am Stat Assoc 47:583-621.

Li D and Zhang X (2002) Physical localization of the 18S$5.8 \mathrm{~S}-26 \mathrm{~S}$ rDNA and sequence analysis of ITS regions in Thinopyrum ponticum (Poaceae, Triticaceae): Implications for concerted evolution. Ann Bot 90:445-452.

Librado P and Rojas J (2009) DnaSP v5: A software for comprehensive analysis of DNA polymorphism data. Bioinformatics 25:1451-1452.

Liu JS and Schardl CL (1994) A conserved sequence in internal transcribed spacer 1 of plant nuclear rRNA genes. Plant Mol Biol 26:775-778.

Lorenz-Lemke AP, Muschner VC, Bonatto SL, Cervi AC, Salzano FM and Freitas LB (2005) Phylogeographic inferences concerning evolution of Brazilian Passiflora actinia and P. elegans (Passifloraceae) based on ITS (nr DNA) variation. Ann Bot 95:799-806.

Mäder G, Zamberlan PM, Fagundes NJR, Magnus T, Salzano FM, Bonatto SL and Freitas LB (2010) The use and limits of ITS data in the analysis of intraspecific variation in Passiflora L. (Passifloraceae). Genet Mol Biol 33:99-108.

Mai JC and Coleman AW (1997) The internal transcribed spacer 2 exhibits a common secondary structure in green algae and flowering plants. J Mol Evol 44:258-271.

Melo NF and Guerra M (2003) Variability of the 5S and 45S rDNA sites in Passiflora L. species with distinct base chromosome numbers. Ann Bot 92:309-316.

Muschner VC, Lorenz AP, Cervi AC, Bonatto SL, Souza-Chies TT, Salzano FM and Freitas LB (2003) A first molecular phylogenetic analysis of Passiflora (Passifloraceae). Am J Bot 90:1229-1238.

Muschner VC, Zamberlan PM, Bonatto SL and Freitas LB (2012) Phylogeny, biogeography and divergent times in Passiflora (Passifloraceae). Genet Mol Biol 35:1036-1043.

Musters W, Boon K, van der Sande CA, van Heerikhuizen H and Planta RJ (1990) Functional analysis of transcribed spacers of yeast ribosomal DNA. EMBO J 9:3989-3996.

Queiroz CS, Batista FRC and Oliveira LO (2011) Evolution of the 5.8S nrDNA gene and internal transcribed spacers in Carapichea ipecacuanha (Rubiaceae) within a phylogeographic context. Mol Phylogenet Evol 59:293-302.

R Development Core Team (2011) R: A language and environment for statistical computing. R Foundation for Statistical Computing. Vienna, Austria. Available online at http://www.R-project.org/.

Ramaiya SD, Bujang JS and Zakaria MH (2014) Genetic diversity in Passiflora species assessed by morphological and ITS sequences analysis. Sci World J 2014:598313.

Rauscher JT, Doyle JJ and Brown AHD (2004) Multiple origins and nrDNA Internal Transcribed Spacer homeologue evolu- 
tion in the Glycine tomentella (Leguminosae) allopolyploid complex. Genetics 166:987-998.

Reuster JS and Mathews DH (2010) RNAstructure: Software for RNA secondary structure prediction and analysis. BMC Bioinformatics 11:129.

Roy A, Frascaria N, Mackay J and Bousquet J (1992) Segregating random amplified polymorphic DNAs (RAPDs) in Betula alleghaniensis. Theor Appl Genet 85:173-180.

Shultz J, Maisel S, Gerlach D, Müller T and Wolf M (2005) A common core of secondary structure of the internal transcribed spacer 2 (ITS2) throughout the Eukaryota. RNA 11:361-364.

Simmons MP and Ochoterena H (2000) Gaps as characters in sequence based phylogenetic analyses. Syst Biol 49:369-381.

Tamura K, Stecher G, Peterson D, Filipski A and Kumar S (2013) MEGA6: Molecular Evolutionary Genetics Analysis version 6.0. Mol Biol Evol 30:2725-2729.

Thompson JD, Gibson TJ, Plewniak F, Jeanmougin F and Higgins DG (1997) The CLUSTALX windows interface: Flexible strategies for multiple sequences alignment aided by quality analysis tools. Nucleic Acids Res 25:4876-4882.

Ulmer T and MacDougal JM (2004) Passiflora - Passionflowers of the World. Timber Press, Portland, $430 \mathrm{p}$.

van der Sande CA, Kwa M, Nues RW van, Heerikhuizen $H$ van, Raué HA and Planta RJ (1992) Functional analysis of internal transcribed spacer 2 of Saccharomyces cerevisiae ribosomal DNA. J Mol Biol 223:899-910.

Wendel JF, Schnabel A and Seelanan T (1995) Bidirectional interlocus concerted evolution following allopolyploid speciation in cotton (Gossypium). Proc Natl Acad Sci U S A 92:280-284.

White TJ, Bruns T, Lee S and Taylor J (1990) Amplication and direct sequencing of fungal ribosomal RNA genes for phylogenetics. In: Innis M, Gelfand D, Sninsky J and White T (eds) PCR Protocols: A Guide to Methods and Applications. Academic Press, San Diego, pp 315-322.

Wissemann V and Ritz CM (2005) The genus Rosa (Rosoideae, Rosaceae) revisited: Molecular analysis of nrITS-1 and $a t p B-r b c L$ intergenic spacers (IGS) versus conventional taxonomy. Bot J Linn Soc Lond 147:275-290.

Yockteng R and Nadot S (2004a) Infrageneric phylogenies: A comparison of chloroplast-expressed glutamine synthetase, cytosol-expressed glutamine synthetase and cpDNA maturase $\mathrm{K}$ in Passißora. Mol Phylogenet Evol. 31:397-402.

Yockteng R and Nadot S (2004b) Phylogenetic relationships among Passiflora species based on the glutamine synthase nuclear gene expressed in chloroplast (ncpGS). Mol Phylogenet Evol 31:379-396.

Young ND and Healy J (2003) GapCoder automates the use of indel characters in phylogenetic analysis. BMC Bioinformatics 4:6.

Zimmer EA, Roalson EH, Skog LE, Boggan JK and Idnurm A (2002) Phylogenetic relationships in the Gesnerioideae (Gesneriaceae) based on nrDNA ITS and cpDNA trnL-F and trnE-T spacer region sequences. Am J Bot 89:296-311.

\section{Supplementary material}

The following online material is available for this article:

Figure S1 - Phylogenetic trees based on ITS sequences for Astrophea, ITS1.

Figure S2 - Phylogenetic trees based on ITS sequences for Astrophea, ITS2.

Figure S3 - Phylogenetic trees based on ITS sequences for Decaloba, ITS1.

Figure S4 - Phylogenetic trees based on ITS sequences for Decaloba, ITS2.

Figure S5 - Phylogenetic trees based on ITS sequences for Deidamioides, ITS1.

Figure S6. Phylogenetic trees based on ITS sequences for Deidamioides, ITS2.

Figure S7. Phylogenetic trees based on ITS sequences for Passiflora, ITS1.

Figure S8 - Phylogenetic trees based on ITS sequences for Passiflora, ITS2.

Table S1 - ITS1 and ITS2 sequences parameters analyzed

Table S2 - Random sequences parameters analyzed. Associate Editor: Marcio de Castro Silva Filho

License information: This is an open-access article distributed under the terms of the Creative Commons Attribution License (type CC-BY), which permits unrestricted use, distribution and reproduction in any medium, provided the original article is properly cited. 